\title{
Intestinal obstruction caused by giant filiform polyposis in a patient with normal colon
}
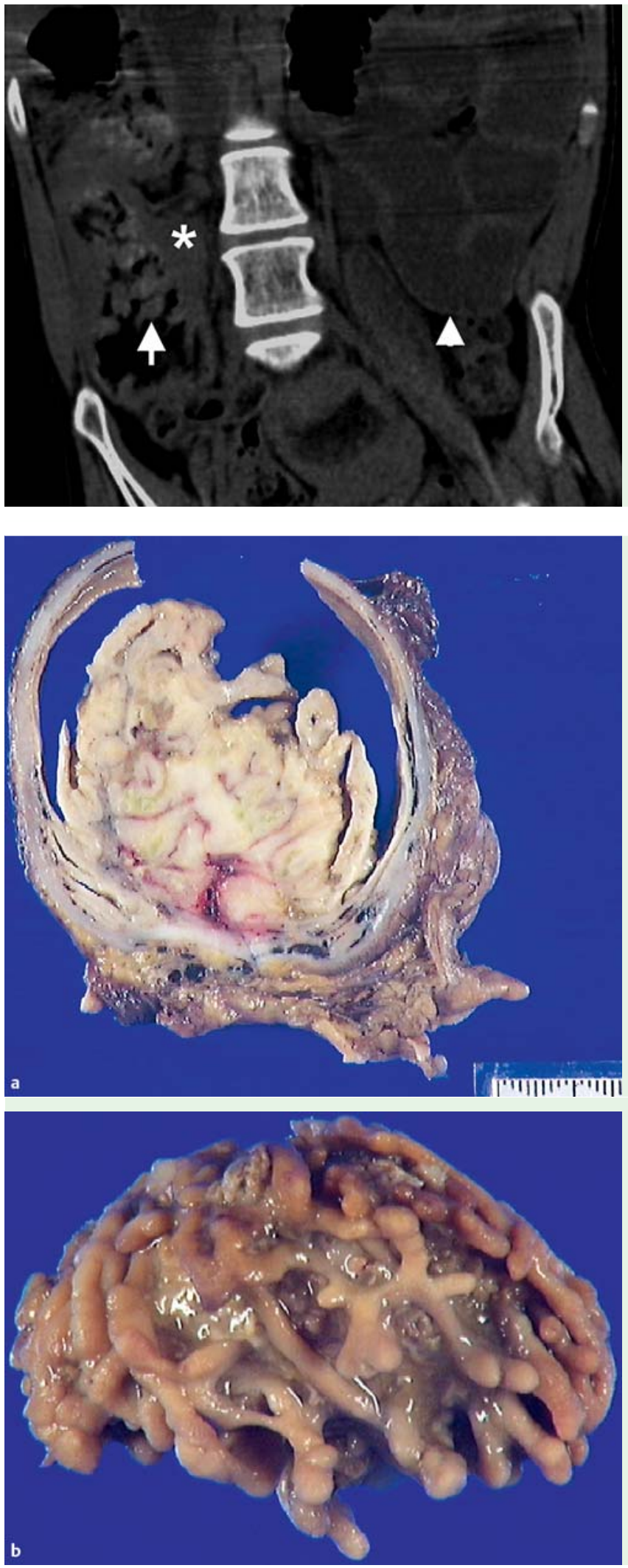

Fig. 1 Computed tomography (CT) in a 31-year-old patient presenting with recurrent episodes of intestinal obstruction but with no previous history of inflammatory bowel disease (IBD). The wall of the right colon is thickened (asterisk) with intraluminal fingerlike lesions (arrow) and dilated small bowel loops (arrowhead).

Fig. 3 a Macroscopic findings of the resected segment. The mass filled the entire lumen of the colon. $\mathbf{b}$ The lesion had a coral reeflike surface appearance with soft, fingerlike protrusions into the lumen.

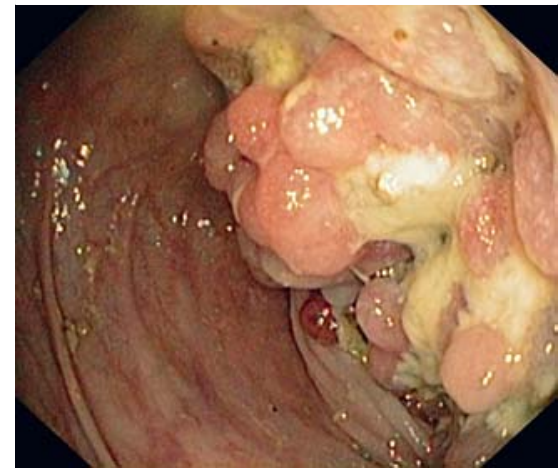

Fig. 2 Colonoscopic view of the polypoid mass obstructing the lumen of the right colon.

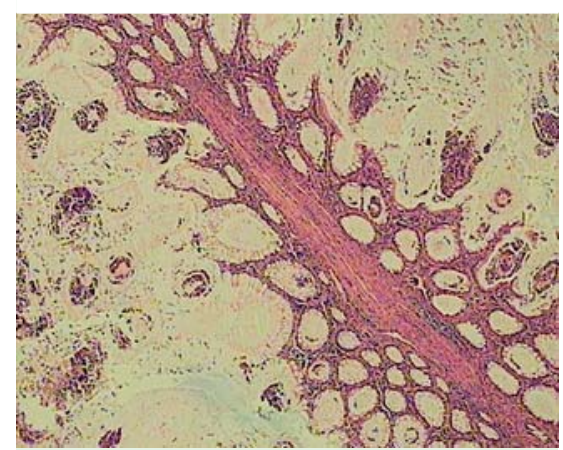

Fig. 4 Histological section showed filiform projections of the mucosa with evidence of chronic inflammation. No crypt abscesses were noted (hematoxylin and eosin staining).

A 31-year-old patient with no previous history of inflammatory bowel disease (IBD) underwent further evaluation for recurrent episodes of intestinal obstruction. Abdominal computed tomography revealed thickening of the wall of the ascending colon and dilated small-bowel loops ( Fig. 1). Colonoscopy disclosed a large polypoid mass with fingerlike projections in the right colon ( $\bullet$ Fig.2). Progression of the endoscope beyond this point was not possible. Biopsies were negative for malignancy. Prompted by the suspicious endoscopic appearance of the lesion and the signs of intestinal obstruction, a right-sided hemicolectomy was carried out. The resected specimen included a mass with multiple clustered polyps with several branches that almost completely obliterated the lumen of the colon ( $\bullet$ Fig.3). Microscopic examination was consistent with giant filiform polyposis ( Fig.4). The nonpolypoid mucosa of 
the terminal ileum and right colon was normal.

Filiform polyposis of the colon is a rare entity, usually encountered in the colon of patients with IBD [1]. However, sporadic cases of filiform polyposis have been reported in patients with histiocytosis X [2], intestinal tuberculosis [3], and ischemic colitis [4]. Filiform polyposis is morphologically characterized by multiple, slender, wormlike projections consisting of submucosal cores lined with normal mucosa. The polyps can range in size from $1.5 \mathrm{~cm}$ to $3 \mathrm{~cm}$ in length and up to $0.5 \mathrm{~cm}$ in diameter [5]. When these polyps adhere to each other, they form large tumorlike masses [1,6-8]. A Pubmed search revealed fewer than 20 reported cases of filiform polyposis without history of IBD [6-9]. In only three of these cases was the lesion circumferential, measuring $4-15 \mathrm{~cm}$ across the largest diameter [6-8]. Filiform polyposis alone is not an indication for resection, but complications such as acute massive hemorrhage, intussusception, and intestinal obstruction may necessitate surgical intervention $[1,6,9,10]$.

Endoscopy_UCTN_Code_CCL_1AD_2AC

\section{Competing interests: None}

\section{G. Mavrogenis', P. Ngendahayo², P. Kisoka' ${ }^{1}$ M. L. Nicholas ${ }^{1}$, E. Kovács ${ }^{3}$, Y. Hoebeke ${ }^{3}$, P. Warzée ${ }^{1}$}

${ }^{1}$ Department of Gastroenterology, Grand Hôpital de Charleroi, Site Notre Dame, Charleroi, Belgium

2 Department of Pathology, Institut de Pathologie et Génétique, Gosselies, Charleroi, Belgium

${ }^{3}$ Department of Digestive Surgery, Grand Hôpital de Charleroi, Site Notre Dame, Charleroi, Belgium

\section{References}

1 Biondi A, Persiani R, Paliani $G$ et al. Giant inflammatory polyposis as the first manifestation of inflammatory bowel disease. Am J Gastroenterol 2009; 104: 2359-2360

2 Hew JM, Chandie Shaw P, Blickman G. Filiform polyposis: a manifestation of histiocytosis X. Fortschr Rontgenstr 1985; 143: 474- 476

3 Peh WC. Filiform polyposis in tuberculosis of the colon. Clin Radiol 1988; 39: 534-536

4 Levine DS, Surawicz CM, Spencer GD et al. Inflammatory polyposis two years after ischemic colon injury. Dig Dis Sci 1986; 31: $1159-1167$

5 Lee CG, Lim YJ, Choi JS et al. Filiform polyposis in the sigmoid colon: a case series. World J Gastroenterol 2010; 16: 2443 - 2447

6 Vainer B, Jess T, Andersen PS. Rapid tumorlike growth of giant filiform polyposis in a patient without a history of chronic bowel inflammation. APMIS 2007; 115: $1306-$ 1310

7 Wolf EM, Strasser C, Geboes K et al. Localized giant inflammatory polyp of the colon in a patient without inflammatory bowel disease. Virchows Arch 2011; 459: 245 - 246

8 Tan KH, Meijer S, Donner R. Giant localized pseudopolyp of the colon without colonic inflammatory disease-case report. Neth J Surg 1987; 39: 95-97

9 Kang HM, Kang YS, Kim SH et al. Single filiform polyp in asymptomatic healthy Korean without history of inflammatory bowel disease. Endoscopy 2007; 39: E333-334

10 Macaigne G, Boivin JF, Cheaib $S$ et al. Single filiform polyp revealed by severe haemorrhage in a patient with normal colon. Report of a case and review of the literature. Gastroenterol Clin Biol 2006; 30: 913-915

\section{Bibliography}

DOI http://dx.doi.org/

10.1055/s-0032-1326264

Endoscopy 2013; 45: E80-E81

(c) Georg Thieme Verlag KG

Stuttgart · New York

ISSN 0013-726X

Corresponding author

G. Mavrogenis

Grand Hôpital de Charleroi

Site Notre Dame

3 Grand Rue

Charleroi 6000

Belgium

Fax: +32-711-02779

mavrogenis@gmail.com 